\title{
La descomposición genética como marco de referencia para el análisis a priori de actividades
}

\section{Genetic decomposition as a frame of reference for a priori analysis of activities}

\author{
Gustavo Daniel Franco Carzolio ${ }^{1}$ \\ Mónica Isabel Olave Baggi² \\ Alejandro Miguel Rosas Mendoza ${ }^{3}$
}

\begin{abstract}
Resumen: En este artículo se presentan los aspectos metodológicos de una investigación basada en la teoría APOE -realizada con estudiantes del profesorado de Matemática de Uruguay-, en donde se estudian fenómenos vinculados a la construcción del esquema de adición de funciones. Particularmente, se centra la atención en el análisis a priori de las actividades que conformaron los cuestionarios utilizados para recabar información. Dicho análisis, basado en las descomposiciones genéticas de adición de funciones, conjunto, función, operación binaria y función suma, permite objetivar y brindar racionalidad a las exigencias cognitivas involucradas en las resoluciones de las distintas actividades propuestas. Lo cual, a su vez, permite apreciar que: (1) las actividades tienen el potencial de capturar un rango amplio del rendimiento matemático de los estudiantes, y (2) los cuestionarios fueron herramientas válidas para la obtención de información con el propósito de responder la pregunta de investigación y alcanzar los objetivos propuestos para el estudio.
\end{abstract}

Fecha de recepción: 7 de agosto de 2020. Fecha de aceptación: 26 de julio de 2021.

1 Instituto de Profesores "Artigas", Departamento de Matemática, Consejo de Formación en Educación, gfrancoc@gmail.com, orcid.org/0000-0002-1677-6810.

2 Instituto de Profesores "Artigas", Departamento de Matemática, Consejo de Formación en Educación, monicaolave23@gmail.com, orcid.org/0000-0001-8231-8036.

3 CICATA-Legaria, IPN, alerosas@ipn.mx, orcid.org/0000-0003-3952-5448. 
Palabras clave: APOE, descomposición genética, esquema, adición de funciones, profesorado de matemática.

\begin{abstract}
This paper presents the methodological aspects of an investigation based on APOS theory-accomplished with prospective middle school mathematics teachers in Uruguay-, in which phenomena related to the construction of functions addition schema are studied. It focuses on the a priori analysis of the questionnaires' activities used for data collection. This analysis is based on the genetic decompositions of functions addition, set, function, binary operation, and sum function, and allows us to objectify and rationalize the cognitive demands involved in solving the offered activities. Furthermore, it makes clear that: (1) a wide range of the students' mathematical performance can be addressed by the activities, and (2) the questionnaires were adequate instruments for gathering information to answer the research question and achieving this study's goals.
\end{abstract}

Keywords: APOS, genetic decomposition, schema, functions addition, prospective middle school mathematics teachers.

\title{
1. INTRODUCCIÓN
}

La investigación que está por detrás de este artículo comienza con dos consideraciones inicialmente desvinculadas: un comentario de la doctora Trigueros (2005, p. 14) y una observación de clase. Trigueros señalaba que los investigadores y los profesores, muchas veces, centran la atención en la enseñanza de conceptos aislados y consideran que los estudiantes serán capaces de establecer relaciones entre ellos; es un problema abierto a la investigación, indicaba la autora, establecer si esto ocurre así en realidad. Por otra parte, habíamos observado que los estudiantes de primer año del profesorado de matemática de Uruguay tenían grandes dificultades para abordar actividades vinculadas al concepto que hemos llamado adición de funciones, a pesar de que con anterioridad se trabajaran, entre otros, los conceptos conjunto, función y operación binaria. Solo teníamos esas dos consideraciones y una incertidumbre: ¿estaban relacionadas?

Hemos denominado adición de funciones a la operación binaria, definida en el conjunto $\mathcal{F}$ de todas las funciones reales, que a cada par ordenado de 
funciones le hace corresponder la función suma -figura 1-. Por otra parte, llamamos funciones reales únicamente a las funciones de dominio y codominio $\mathbb{R}$, y nos centramos en este tipo de funciones para que la determinación del dominio de la función suma no constituya un factor de dificultad para los estudiantes que nos aleje de los objetivos de nuestra investigación.

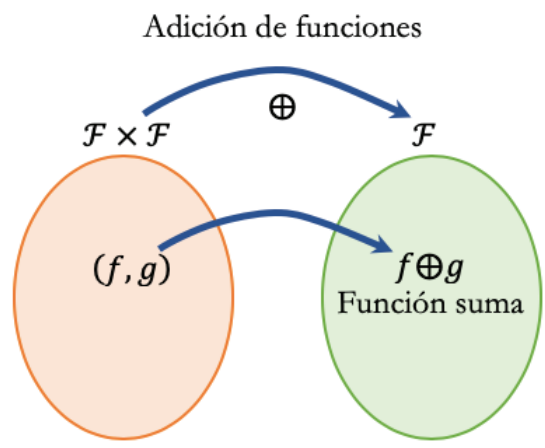

Figura 1 Representación de la adición de funciones

Lo señalado por Trigueros (2005) sugiere -acorde a la observación de clase mencionada anteriormente- que podría no resultar sencillo vincular e integrar diferentes conceptos para abordar actividades relacionadas con la adición de funciones, cuando dicha operación binaria no ha sido trabajada explícitamente en la clase de matemática. Las consideraciones anteriores nos condujeron a interesarnos por analizar la construcción del esquema de adición de funciones, en particular a estudiar fenómenos vinculados a dicha construcción.

A lo largo del estudio, los objetivos y la pregunta de investigación fueron cambiando hasta alcanzar la forma definitiva que presentan a continuación:

- Establecer si es suficiente - para construir el esquema de adición de funciones- abordar en la enseñanza, en forma aislada, conceptos como conjunto, función y operación binaria.

- Establecer fenómenos inherentes a la construcción del esquema de adición de funciones. 
En concordancia con los objetivos, la pregunta de investigación que nos propusimos responder fue la siguiente: ¿Cómo un individuo podría llegar a construir el esquema de adición de funciones? Hay quienes consideran que las modificaciones que se le imprimen a la pregunta de investigación y a los objetivos es a fin de lograr la coherencia interna que necesita toda investigación. En nuestro estudio, las diversas modificaciones que han sufrido la pregunta de investigación y los objetivos, en la búsqueda de coherencia interna, nos permitió encontrar la escultura debajo del exceso pétreo.

Hemos querido abordar el problema de la construcción del esquema de adición de funciones desde un punto de vista complejo (es decir, intentando comprender el entramado en su conjunto, en lugar de fragmentarlo en jirones para su análisis), intentado evitar reducciones que simplificaran ingenuamente el problema. El constructo de esquema de la teoría APOE habilita este tipo de abordaje, no solo porque los esquemas contienen las descripciones, la organización y las ejemplificaciones de las estructuras mentales que un individuo ha construido en torno a un concepto matemático (Arnon et al., 2014, p. 25), sino porque para comprender el desarrollo de un esquema es necesario analizar cómo se coordina con otros esquemas relacionados (p. 122). Por este motivo la noción de esquema fue central en nuestra investigación.

\section{LA TEORÍA APOE}

La teoría APOE - desarrollada por Ed Dubinsky a principios de los años ochenta a partir del trabajo de Jean Piaget- nos brindó un marco de referencia idóneo para llevar adelante nuestra investigación, fundamentalmente por dos motivos: (1) porque permite comprender cómo un individuo construye, desde un punto de vista cognitivo, los conceptos matemáticos (particularmente los correspondientes a la enseñanza superior), y (2) porque es especialmente adecuada para trabajar con el concepto función (concepto clave en nuestro estudio, dado que la adición de funciones es un caso particular de función que, además, tiene como dominio el conjunto formado por todos los pares ordenados de funciones reales y como codominio el conjunto de todas las funciones reales):

En particular, la formulación de Dubinsky demuestra ser especialmente adecuada para el concepto función porque la función es a la vez un proceso (entrada-salida) y un objeto matemático que necesita ser tratado como una entidad conceptual... Por 
lo tanto, la técnica de encapsulación del proceso de función en un objeto matemático parece ser una combinación perfecta para el desarrollo del concepto función. (Eisenberg, 1991, p. 143)

\subsection{LAS ESTRUCTURAS MENTALES}

Como lo evidencian los objetivos y la pregunta de investigación, es central para nuestro estudio la noción de esquema. Esta noción forma parte de las denominadas -en la teoría APOE- estructuras mentales, junto con las acciones, los procesos y los objetos. Los individuos, para dar sentido a una situación matemática, construyen y utilizan acciones, procesos y objetos y los organizan en esquemas. Estas estructuras mentales son consideradas, en la teoría APOE, como etapas en el aprendizaje de los conceptos matemáticos.

Una acción es una transformación de objetos percibidos por el individuo como esencialmente externos y que requiere, ya sea explícitamente o desde la memoria, instrucciones paso a paso sobre cómo realizar la operación...

Cuando una acción se repite y el individuo reflexiona sobre ella, él o ella pueden hacer una construcción mental interna llamada proceso con lo cual el individuo puede pensar en realizar el mismo tipo de acción, pero ya no con la necesidad de estímulos externos...

Un objeto se construye a partir de un proceso cuando el individuo toma conciencia del proceso como una totalidad y se da cuenta de que las transformaciones pueden actuar sobre este...

Por último, un esquema para un cierto concepto matemático es una colección personal de acciones, procesos, objetos y otros esquemas que están vinculados por algunos principios generales para formar un marco de referencia en la mente del individuo que puede ser traído para afrontar una situación problemática que involucra al concepto. (Dubinsky y McDonald, 2001, pp. 276-277)

Para ejemplificar las distintas estructuras mentales descritas anteriormente, consideremos el concepto función. Una persona que requiere una expresión algebraica para trabajar con una función y puede hacer poco más que sustituir la variable por valores particulares del dominio, se considera que tiene una concepción acción de función. Un individuo con una concepción proceso pensará a la función en términos de elementos de entrada que se transforman para 
producir elementos de salida. Una concepción objeto permite al individuo aplicar transformaciones a las funciones y concebir, por ejemplo, un conjunto de funciones, definir operaciones en dicho conjunto, equiparlo con una topología, etc. El esquema de función puede estar compuesto por diferentes tipos de funciones, como funciones de variable real, funciones de varias variables o funciones proposicionales. Estos diferentes tipos de funciones pueden haberse construido como procesos u objetos (Arnon et al., 2014).

Por último, y volviendo a la estructura mental que ocupa un lugar privilegiado en nuestro estudio, una característica importante del esquema es la coherencia, la cual queda establecida por la capacidad del individuo para determinar qué fenómenos están al alcance del esquema y cuáles no.

\subsection{DESCOMPOSICIÓN GENÉTICA}

Para cada concepto particular, las estructuras mentales son descritas a través de un modelo hipotético llamado descomposición genética. Una descomposición genética es un modelo epistemológico y cognitivo de un concepto matemático en el que se describen las estructuras y los mecanismos mentales que un individuo necesitaría construir para aprender dicho concepto. Hasta que se pruebe experimentalmente, una descomposición genética es una hipótesis y se conoce como preliminar. Una descomposición genética preliminar está basada en la compresión del concepto por parte de los investigadores, sus experiencias de aula en torno al mismo, investigaciones previas que informen sobre las concepciones de los estudiantes, perspectivas históricas sobre su desarrollo, y análisis de textos y materiales de enseñanza relacionados con dicho concepto. (Arnon et al., 2014, pp. 27-28)

\subsection{CICLO DE INVESTIGACIÓN}

La teoría APOE incluye un ciclo de investigación que está formado por tres componentes: análisis teórico, diseño e implementación de un plan de enseñanza, y recopilación y análisis de datos. De acuerdo con esta teoría, la investigación comienza con un análisis teórico y cognitivo del concepto matemático considerado, lo cual da lugar a una descomposición genética preliminar de dicho concepto. A partir de este análisis, se diseña e implementa un plan de enseñanza con la finalidad de que los estudiantes realicen las construcciones mentales establecidas en la descomposición genética preliminar. Durante la enseñanza 
se recolectan datos, los que luego serán analizados utilizando como marco teórico la teoría APOE, con la finalidad de responder la pregunta de investigación y alcanzar los objetivos propuestos para el estudio.

Para dicha finalidad, no solo se tienen en cuenta los datos recogidos durante la enseñanza, también se pueden incluir cuestionarios, entrevistas semiestructuradas (grabadas en audio o video), pruebas y el trabajo con computadoras.

Las entrevistas son los medios más importantes por los cuales se recopilan los datos en las investigaciones basadas en APOE...

Los individuos entrevistados se pueden seleccionar a partir de sus respuestas a un cuestionario escrito o a un examen previamente administrado, retroalimentación del instructor o una combinación de esos criterios. (Arnon et al., 2014, p. 95)

Para intentar responder nuestra pregunta de investigación, hemos propuesto dos cuestionarios a estudiantes de primer año del profesorado de matemática de Uruguay. Luego de propuesto cada cuestionario, realizamos un análisis preliminar de las respuestas de los estudiantes a partir del cual seleccionamos algunos para realizarles entrevistas. En nuestro estudio las entrevistas jugaron un papel fundamental: la complejidad y sutileza de los conceptos matemáticos abordados en nuestra investigación, y los objetivos que nos propusimos, hizo de las entrevistas el medio más importante de recolección de información. A través de preguntas minuciosas fue posible, por ejemplo, detectar fenómenos inherentes a la construcción del esquema de adición de funciones; fenómenos que habían pasado desapercibidos en las respuestas a las preguntas de los cuestionarios.

Por otra parte, y volviendo al ciclo de investigación de la teoría APOE, Roa-Fuentes y Oktaç (2012, pp. 204-205) señalan:

Aspectos como este, relacionados con el tiempo y el número de integrantes del equipo de investigadores necesarios para hacer el seguimiento de grupos de estudiantes, han generado la realización de investigaciones que pasan de la primera a la tercera componente. Como es el caso del trabajo que presentamos en este escrito, una vez se tiene la descomposición genética preliminar, se diseñan instrumentos como pruebas diagnósticas y entrevistas para analizar la manera como los individuos han construido o están construyendo un concepto. Esto permite que aún sin haber desarrollado un proceso de enseñanza, se analice la viabilidad de los aspectos puramente teóricos de la descomposición genética preliminar. 
En nuestro estudio también omitimos el segundo componente, pero, más que por las dificultades técnicas de la aplicación del ciclo de investigación, fue debido a la finalidad de este. Nuestro propósito no era que los estudiantes realizaran las construcciones mentales requeridas por la descomposición genética preliminar de adición de funciones, nuestro interés estaba centrado en aportar información sobre la construcción del esquema de adición de funciones: ¿ipueden los estudiantes que participaron en nuestra investigación, por ellos mismos (sin que medie ningún plan de enseñanza), establecer vínculos entre conceptos como conjunto, función y operación binaria para resolver actividades vinculadas con la adición de funciones y comenzar a estructurar el esquema de dicho concepto?

Por último, el análisis de los datos recabados, correspondiente al tercero de los componentes del ciclo de investigación, refina (o valida) la descomposición genética preliminar, de modo que sea más congruente con la manera en que los estudiantes construyen el conocimiento. En nuestra investigación, el análisis de la información recogida a través de los cuestionarios y las entrevistas nos permitió validar la descomposición genética preliminar de adición de funciones y refinar la descomposición genética preliminar de función suma. Estas descomposiciones genéticas (la validada y la refinada) fueron utilizadas luego como marco de referencia para detectar fenómenos vinculados a la construcción del esquema de adición de funciones.

\section{ASPECTOS METODOLÓGICOS}

Nuestro estudio comienza con una descomposición genética preliminar de adición de funciones. Luego, a partir de un análisis de la concepción proceso de adición de funciones, establecimos que los esquemas de conjunto, función, operación binaria y función suma son componentes del esquema de adición de funciones. Por lo tanto, debido a los objetivos de nuestro estudio, fue necesario contar con descomposiciones genéticas de conjunto, función, operación binaria y función suma. Para los tres primeros conceptos no fue necesario realizar descomposiciones genéticas, dado que ya existen en la literatura y están recogidas en Arnon et al. (2014); en cambio fue necesario elaborar una descomposición genética preliminar de función suma. Estas descomposiciones genéticas fueron utilizadas, inicialmente, para realizar un análisis a priori de las actividades de los cuestionarios, con el propósito de validar dichos cuestionarios como herramientas 
idóneas para la obtención de información que nos permitiera responder nuestra pregunta de investigación y alcanzar los objetivos propuestos para el estudio.

\subsection{DESCOMPOSICIÓN GENÉTICA PRELIMINAR DE ADICIÓN DE FUNCIONES}

A partir de la descomposición genética de operación binaria presente en Arnon et al. (2014, p. 48), de nuestra comprensión del concepto adición de funciones y de nuestra experiencia de aula en torno al mismo, hemos realizado la descomposición genética preliminar de adición de funciones que presentamos a continuación.

Acción: $\quad$ un individuo presenta una concepción acción si, dadas dos funciones reales $f$ y $g$ particulares a través de sus expresiones analíticas, puede realizar poco más que manipulaciones algebraicas sobre la expresión analítica de $f \oplus g$.

Proceso: $\quad$ un individuo muestra una concepción proceso si puede concebir a la adición de funciones como la función que a todo par ordenado $(f, g)$ de funciones reales le hace corresponder la función suma $f \oplus g$.

Objeto: $\quad$ un individuo evidencia una concepción objeto si puede distinguir entre la adición de funciones y otras operaciones binarias definidas en $\mathcal{F}$ (conjunto de todas las funciones reales); puede hallar similitudes y diferencias entre la adición de funciones y otras operaciones binarias (por ejemplo la adición de reales); puede desencapsular la adición de funciones y reconocer, en el proceso resultante, a la función suma como el resultado de operar dos funciones reales según la adición de funciones; y puede encapsular el proceso para considerar, por ejemplo, la estructura $(\mathcal{F}, \oplus)$.

Esquema: un individuo tiene un esquema para la adición de funciones si posee una colección de acciones, procesos y objetos vinculados a dicho concepto, que surgen como consecuencia de una interrelación entre sus esquemas componentes.

\subsection{ESQUEMAS COMPONENTES DEL ESQUEMA DE ADICIÓN DE FUNCIONES}

Debido a que estamos interesados en la construcción del esquema de adición de funciones, es necesario tener en cuenta que el desarrollo de un esquema depende a su vez de otros esquemas:

En el proceso de aprendizaje, a medida que se desarrolla el conocimiento, un individuo puede construir Esquemas coexistentes que cambian constantemente y que están en distintas etapas de desarrollo. Cada Esquema está compuesto por Acciones, 
Procesos, Objetos y otros Esquemas y por las relaciones entre esas estructuras. Al enfrentar una situación problemática, una persona puede necesitar coordinar diferentes esquemas. Uno de los objetivos de la investigación es identificar los diferentes Esquemas que necesitan ser desarrollados y cómo son coordinados o cómo interactúan. Por lo tanto, para comprender el desarrollo de un Esquema, la investigación no solo debe determinar cómo se construye el Esquema sino cómo se puede coordinar con otros Esquemas relacionados. (Arnon et al., 2014, p. 122)

Por lo tanto, con la finalidad de responder nuestra pregunta de investigación - ¿Cómo un individuo podría llegar a construir el esquema de adición de funciones?-, nos propusimos identificar diferentes esquemas que interactúan en el desarrollo del esquema de adición de funciones. Un análisis de la adición de funciones como proceso (que presentamos a continuación) nos permitió establecer que los esquemas de conjunto, función, operación binaria y función suma son componentes del esquema de adición de funciones.

\subsection{LA ADICIÓN DE FUNCIONES COMO PROCESO}

Consideremos la adición de funciones $(\oplus)$ definida en el conjunto formado por todas las funciones reales $(\mathcal{F})$. Para desencapsular el objeto adición de funciones y pasarlo a considerar como un proceso, es necesario una concepción proceso de conjunto (que permita desencapsular el objeto $\mathcal{F}$ ) de modo que sea posible considerar dos funciones reales $f$ y $g$ cualesquiera -como objetos-; y también una concepción proceso de operación binaria que habilite la búsqueda del correspondiente del par ordenado $(f, g)$ a través de la adición de funciones. A continuación, se deben desencapsular las funciones $f$ y $g$ (objetos) para concebirlas como los procesos $f(x)$ y $g(x)$, para todo real $x$. Estos procesos deben ser coordinados a través de la adición de reales para obtener así el proceso $f(x)+g(x)$, para todo real $x$; lo cual conduce a una concepción proceso de función suma. Su encapsulación permite una concepción objeto de función suma. Por último, una concepción proceso de conjunto haría posible identificar la función suma como un elemento del conjunto $\mathcal{F}$ (figura 2). 


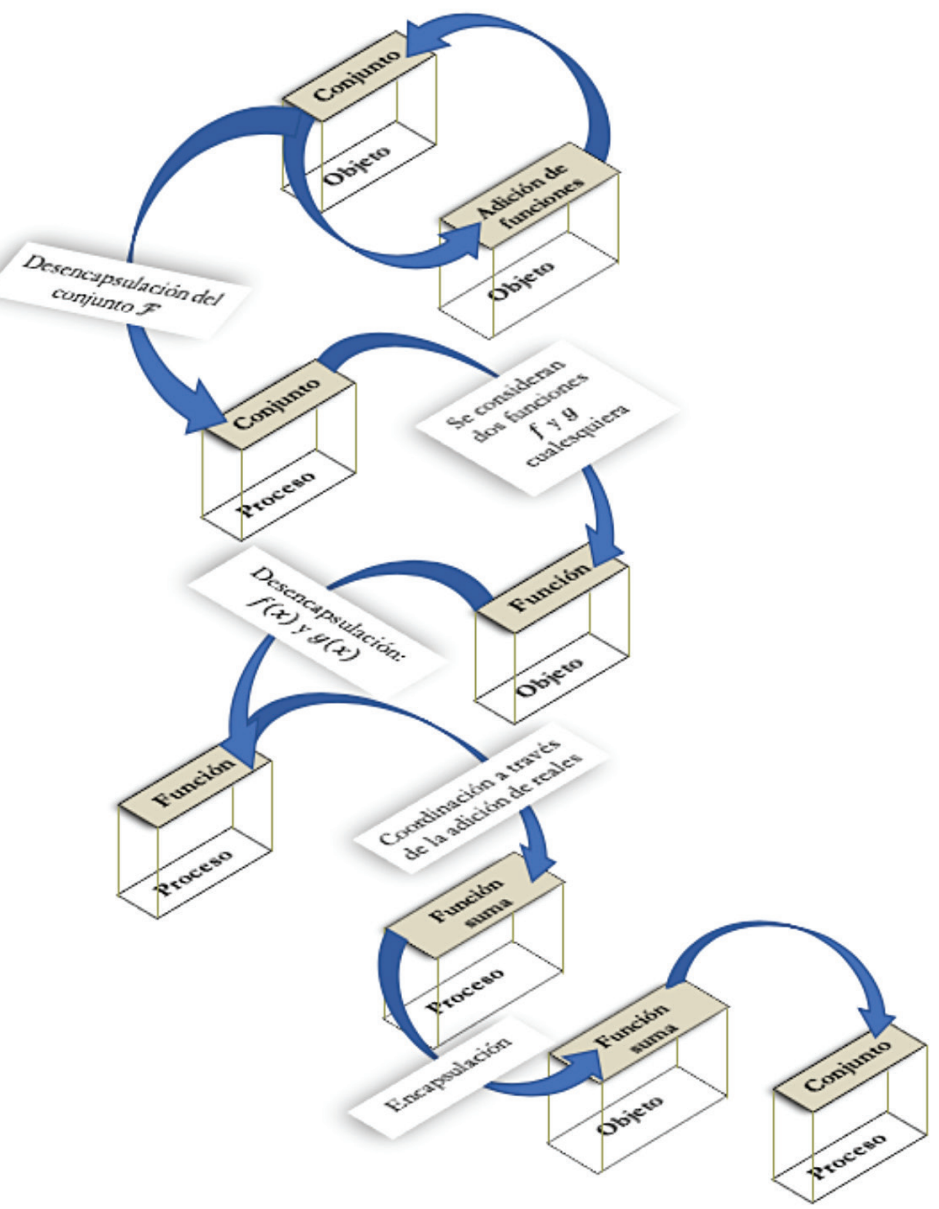

Figura 2. La adición de funciones como proceso.

\subsection{DESCOMPOSICIONES GENÉTICAS DE CONCEPTOS CUYOS ESQUEMAS SON COMPONENTES DEL ESQUEMA DE ADICIÓN DE FUNCIONES}

Según el análisis presentado anteriormente, una concepción proceso de adición de funciones requiere concepciones objeto y proceso de función y de función suma, y concepciones proceso de conjunto y operación binaria. Por lo tanto, podemos establecer que los esquemas de conjunto, función, función suma y operación 
binaria son componentes del esquema de adición de funciones. Debido al propósito de nuestra investigación, fue relevante entonces no solo contar con una descomposición genética de adición de funciones, sino con descomposiciones genéticas de conjunto, función, función suma y operación binaria. No fue necesario realizar descomposiciones genéticas de conjunto, función y operación binaria dado que ya existen en la literatura (Arnon et al., 2014). En cambio, realizamos una descomposición genética preliminar de función suma a partir de la descomposición genética de función, de nuestro conocimiento disciplinar y de nuestra experiencia de aula.

A continuación, presentamos las descomposiciones genéticas de conjunto, función y operación binaria que aparecen en Arnon et al. (2014). Luego, proponemos la descomposición genética preliminar de función suma.

\subsubsection{Descomposición genética de conjunto}

En Arnon et al. (2014, pp. 48-49) se presenta la siguiente descomposición genética del concepto conjunto:

Acción: $\quad$ un individuo solo puede concebir un conjunto cuando se le brinda una lista específica de elementos o cuando se le presenta una condición particular de pertenencia al conjunto.

Proceso: $\quad$ se interioriza la acción de reunir y juntar objetos en una colección según alguna condición.

Objeto: el individuo puede aplicar acciones o procesos al proceso, como determinar la cardinalidad de un conjunto, comparar dos conjuntos (no necesariamente en términos de cardinalidad), considerar un conjunto como un elemento de otro conjunto y definir una función en la que un conjunto es uno de los elementos del dominio. El individuo también está en condiciones de desencapsular un conjunto para que pueda coordinarse con otros procesos.

Esquema: el individuo puede aplicar el esquema de conjunto a una situación matemática dada. En álgebra lineal, esto significaría poder definir conjuntos de objetos que luego podrían clasificarse como conjuntos de vectores: polinomios, funciones y matrices. Un individuo también puede haber desarrollado una noción general acerca de lo que es un conjunto y de lo que no es. 


\subsubsection{Descomposición genética de función}

La descomposición genética del concepto función presente en Arnon et al. (2014, pp. 29-30) y basada en las ideas de Dubinsky, es la siguiente:

Acción: dado un conjunto de números (u otro tipo de elementos), se realizan acciones sobre este conjunto. Estas acciones implican tomar un elemento de dicho conjunto y aplicar una regla explícita -generalmente una fórmula algebraica- y asignar a ese elemento específico un único elemento de un segundo conjunto.

Proceso: $\quad$ un individuo que muestra una concepción proceso de función puede pensar una función en términos de tomar entradas, manipularlas de algún modo, y producir salidas, sin la necesidad de hacer cálculos explícitos. Una concepción proceso de función puede incluir la capacidad de determinar si una función tiene inversa (lo que requeriría una inversión del proceso función) o de describir cómo se podría componer dos funciones (lo que requeriría una coordinación de dos procesos).

Objeto: $\quad$ las acciones u otros métodos aplicados al proceso función conducen a su encapsulación como un objeto cognitivo. A través del mecanismo de encapsulación el individuo pasa de percibir a la función como una transformación dinámica a concebirla como una entidad estática que puede ser examinada y transformada. La encapsulación le permitiría al individuo concebir conjuntos de funciones, realizar operaciones aritméticas con funciones, o pensar una función como el límite de una sucesión de funciones.

Esquema: un individuo que puede determinar si una relación entre dos conjuntos es función, y puede coordinar varios procesos para hallar el dominio y el recorrido (rango) de una función, estaría evidenciando la construcción del esquema de función. Un indicador de la coherencia de dicho esquema incluiría la capacidad del individuo para determinar si una situación matemática particular puede ser modelada a través de una función.

\subsubsection{Descomposición genética de operación binaria}

En Arnon et al. (2014, p. 48) se señala que una operación binaria es una función de dos variables y se presenta la siguiente descomposición genética de este concepto: 
Acción: dada una fórmula para una operación binaria, un individuo puede tomar dos elementos específicos del conjunto, en el que se define la operación, y aplicar la fórmula.

Proceso: el individuo interioriza las acciones vinculadas a una operación binaria: toma dos elementos, actúa sobre estos objetos de algún modo, y devuelve un objeto nuevo.

Objeto: $\quad$ el individuo puede distinguir entre dos operaciones binarias, considerar más de una operación binaria definida en un conjunto, verificar si una operación binaria satisface una propiedad y desencapsular una operación binaria para que pueda coordinarse con otros procesos.

Esquema: el individuo puede definir una operación binaria en un conjunto e identificar si una relación es una operación binaria.

\subsubsection{Descomposición genética preliminar de función suma}

\section{Debido a que no hemos encontrado en la literatura una descomposición gené- tica del concepto función suma, a continuación, presentamos una descomposi- ción genética preliminar de dicho concepto.}

Acción: $\quad$ un individuo tiene una concepción acción de función suma si, dadas dos funciones reales $f$ y $g$, puede hacer poco más que tomar un número real específico y aplicar una regla explícita -generalmente una fórmula algebraica, pero también podría extraer la información de una tabla- para encontrar su imagen según $f$ y su imagen según $g$ y, luego, sumando dichas imágenes, obtener la imagen del real según la función suma.

Proceso: $\quad$ un individuo muestra una concepción proceso de función suma si puede pensar a la función $f \oplus g$ como la función que a todo real, arbitrario, le hace corresponder la suma de las imágenes según $f$ y $g$.

Objeto: $\quad$ un individuo evidencia una concepción objeto de función suma si la puede considerar como una nueva función objeto: a través del mecanismo de encapsulación el individuo pasa de concebir a la función suma como una transformación dinámica a concebirla como una entidad estática que puede ser examinada y transformada. La encapsulación le permitiría concebir, por ejemplo, a la función suma como elemento de un conjunto. Por otra parte, el individuo debe poder desencapsular el objeto función suma y reconocer, en el proceso resultante, la coordinación de dos procesos a través de la adición de reales.

Esquema: el esquema de función suma puede estar compuesto por distintas funciones suma $f \oplus g$ : la función $f$ podría ser polinómica, racional, trigonométrica, etc. Por otra parte, podría cumplir distintas propiedades: podría ser continua (o no), derivable (o no), etc. Lo mismo ocurre con la función $g$. 


\subsection{SOBRE LA RECOPILACIÓN Y EL ANÁLISIS DE LOS DATOS}

En esta subsección presentamos, en primer lugar, aspectos generales vinculados con la población y con los instrumentos que utilizamos para recabar información (cuestionarios y entrevistas). En segundo lugar, proponemos un análisis a priori de las actividades que conformaron los cuestionarios. Dicho análisis se realizó en forma previa a proponer los cuestionarios a los estudiantes (utilizando -únicamente- como marco de referencia las descomposiciones genéticas presentadas anteriormente), pero no fue el primero de los análisis realizados. La selección, elaboración y reelaboración de las actividades conlleva un análisis que determina la pertinencia y adecuación de las actividades propuestas en relación con los objetivos y con la pregunta de investigación. En otras palabras, el análisis a priori no recoge el proceso de elaboración de los cuestionarios sino más bien es un análisis que justifica, posteriormente a dicha elaboración, el potencial que tienen unas ciertas actividades para recabar información valiosa en relación con nuestra investigación.

\subsubsection{Aspectos generales sobre la población y los instrumentos}

Para recabar información que nos permitiera responder la pregunta de investigación y alcanzar los objetivos propuestos para el estudio, elaboramos un cuestionario (cuestionario 1) compuesto por siete actividades. A continuación, realizamos un análisis a priori de dichas actividades en términos de la teoría APOE, para lo cual utilizamos como marco de referencia las descomposiciones genéticas que existen en la literatura de conjunto, función y operación binaria (Arnon et al., 2014), y las descomposiciones genéticas preliminares de adición de funciones y de función suma que presentamos anteriormente.

Este primer cuestionario fue propuesto en 2018 a un grupo integrado por 24 estudiantes de primer año del profesorado de matemática de Uruguay -del curso Fundamentos de la Matemática, del cual uno de los autores de este artículo era el profesor-, luego que los conceptos conjunto, función y operación binaria, habían sido abordados. Si bien en la actividad 1 aparecía la definición de función suma, como queríamos que los estudiantes tuvieran acceso a ella a lo largo de todo el cuestionario, una vez retirada la actividad 1, y antes de entregar la actividad 2, les proporcionamos nuevamente la definición de función suma escrita en una hoja. En la definición que le damos luego de retirada la actividad 1 hay una mayor presencia del lenguaje natural, debido a que queríamos evitar 
que una falta de comprensión de la definición les impidiera la resolución de las actividades. De todas formas, no prescinde totalmente del lenguaje simbólico: tratamos de encontrar un justo equilibrio entre el lenguaje natural y el simbólico. El lenguaje natural puede resultar más cercano a los estudiantes, pero puede convertirse en un trabalenguas que vacíe de significado la definición. El lenguaje simbólico tiene la virtud de la concisión, que puede redundar en claridad, pero es necesario tener las herramientas para una adecuada comprensión. El tiempo aproximado que demandó la realización del cuestionario 1 fue una hora y media.

Luego de propuesto el cuestionario realizamos un análisis preliminar de las respuestas de los estudiantes, para el cual tuvimos en cuenta el análisis a priori de las actividades. Este análisis preliminar nos permitió seleccionar seis estudiantes, en un amplio rango de rendimiento matemático, para realizarles entrevistas, con la finalidad de obtener información que nos permitiera comprender cómo habían articulado su pensamiento para brindar respuesta a cada una de las actividades.

A partir del análisis de las respuestas a las preguntas del cuestionario y de las entrevistas, comenzó una primera etapa de teorización. En esta primera etapa observamos un fenómeno al que denominamos identificación.

La adición de funciones $(\oplus)$-como proceso- es la operación binaria que a cada par ordenado de funciones reales $(f, g)$ le asigna como resultado la función suma $f \oplus g$. Observamos en las respuestas a las preguntas del cuestionario y de las entrevistas que, con frecuencia, los estudiantes identificaban la adición de funciones con la función suma, de modo que consideraban a ambos conceptos como si fueran el mismo. Esto puede evidenciarse en las siguientes respuestas a actividades del cuestionario:

$g \oplus h$ es una operación binaria definida en $\mathcal{F}$ [conjunto formado por funciones] porque cumple clausura y unicidad, el resultado de operar va a pertenecer a $\mathcal{F}$ y será único. [Respuesta de Celeste a una actividad del cuestionario.]

$\oplus$ es op. binaria definida en $\mathbb{R} \rightarrow \mathbb{R}$. [Respuesta de Rosalía a una actividad del cuestionario.]

Celeste indica que $g \oplus h$ es una operación binaria, lo cual es incorrecto puesto que la función suma es una función de $\mathbb{R}$ en $\mathbb{R}$. Rosalía señala que $\oplus$ es una 
función de $\mathbb{R}$ en $\mathbb{R}$, sin embargo $\oplus$ es una relación de $\mathcal{F} \times \mathcal{F}$ en $\mathcal{F}$; en cambio $g \oplus h$ sí es una función de $\mathbb{R}$ en $\mathbb{R}$.

Lo inesperado del fenómeno llamó tanto nuestra atención que decidimos proponer en 2019, a otro grupo integrado por 27 estudiantes de primer año del profesorado de matemática de Uruguay (nuevamente del curso Fundamentos de la Matemática), un nuevo cuestionario (cuestionario 2) con la finalidad de rebatir, confirmar o ampliar las observaciones realizadas a partir del primer cuestionario y del primer grupo de entrevistas. El cuestionario 2 estuvo compuesto por tres actividades: la primera coincide, esencialmente (desde el punto de vista matemático), con la actividad 6 del primer cuestionario, y las actividades 2 y 3 no aparecen en el cuestionario 1. Antes de proponer el cuestionario entregamos a los estudiantes una hoja con las definiciones de función, operación binaria y función suma. Si bien las dos primeras ya habían sido trabajadas en el curso de Fundamentos, no queríamos que sus respuestas estuvieran condicionadas por el olvido. El tiempo aproximado que demandó la realización del cuestionario 2 fue 45 minutos.

Lo más importante a destacar del nuevo cuestionario es el cambio deliberado en algunas notaciones: la función suma se notó con la letra $s$, sustituyendo la notación $f \oplus g$, y la adición de funciones se representó con $*$, sustituyendo al símbolo $\oplus$. Estas modificaciones se realizaron con la finalidad de independizar (desde el punto de vista simbólico) la adición de funciones de la función suma, lo cual era clave para establecer si la identificación entre ambos conceptos permanecía incluso cuando no había símbolo que conectara ambos conceptos: en el primer cuestionario el símbolo $\oplus$ era utilizado tanto en la notación de la función suma como para notar la adición de funciones, por lo que la identificación podría haber estado sugerida implícitamente por las notaciones.

Una vez realizado un análisis preliminar de las respuestas al cuestionario 2, seleccionamos siete estudiantes para realizarles entrevistas y profundizar en sus respuestas.

\subsubsection{Análisis a priori de las actividades de los cuestionarios}

Luego de elaborados los cuestionarios realizamos un análisis a priori -en términos de la teoría APOE- de las actividades que los conforman. Este análisis fue realizado a partir de las descomposiciones genéticas que aparecen en Arnon et al. (2014) de conjunto, función y operación binaria y, también, de las descomposiciones genéticas preliminares de adición de funciones y de función suma 
que presentamos más arriba. Este análisis a priori sirvió de referencia para el análisis preliminar de las respuestas de los estudiantes a los cuestionarios y, en consecuencia, para seleccionar los estudiantes que entrevistamos. A continuación, presentaremos cada una de las actividades de sendos cuestionarios y su correspondiente análisis.

\subsubsection{Análisis a priori de las actividades del cuestionario 1}

Presentamos una a una las actividades del cuestionario 1 y su correspondiente análisis a priori.

Actividad 1

Consideremos dos funciones $f: \mathbb{R} \rightarrow \mathbb{R}$ y $g: \mathbb{R} \rightarrow \mathbb{R}$. Se define la función suma de $f$ y $g$, y se la nota $f \oplus g$, de la siguiente manera:

$f \oplus g: \mathbb{R} \rightarrow \mathbb{R}$ tal que $(f \oplus g)(x)=f(x)+g(x)$.

Si tuvieras que explicarle con tus palabras a un compañero cuál es la función suma de las funciones $f$ y $g$, ¿qué le dirías?

La función suma, en esta actividad, podría ser descrita como un objeto, en cuyo caso no hay mucho para decir más que es una función real. También podría ser descrita como un proceso: la función suma $f \oplus g$ es la función que a cada número real le hace corresponder la suma de las imágenes según $f$ y $g$. 0 también a través de una concepción acción: consideremos las funciones reales $f: f(x)=-2 x+5$ y $g: g(x)=3 x^{2}-2 x+1$. La función suma es la función que, por ejemplo, a 1 le asigna como imagen:

$$
f(1)+g(1)=(-2 \cdot 1+5)+\left(3 \cdot 1^{2}-2 \cdot 1+1\right)=5 \text {. }
$$

Como en esta actividad se pone al estudiante ante la situación de tener que explicar a un compañero cuál es la función suma, las respuestas podrían aportar información acerca de cuál considera (implícitamente), un estudiante de profesorado, que es la concepción (en términos de acciones, procesos u objetos) más adecuada para dar una descripción de la función suma; de todas formas esta consideración por parte del estudiante estará subordinada al tipo de concepción que él haya construido. 
Actividad 2

¿Por qué en la definición de función suma aparecen dos signos de "más" distintos: $\oplus y+$ ?

Es posible que algunos estudiantes - a través de una concepción objeto de operación binaria- identifiquen el símbolo "+" con la adición definida en algún conjunto numérico. Otros estudiantes podrían vincular el símbolo " + " con la producción de un "resultado" -lo cual estaría vinculado con una concepción acción de operación binaria-. En relación con el símbolo “ $\bigoplus$ ”, podrían considerarlo -a través de una concepción objeto de función suma- como parte del símbolo utilizado para notar la función suma $f \oplus g$ (sin ningún significado autónomo), o podrían considerarlo como el símbolo utilizado para "sumar funciones" - Io cual estaría vinculado con una concepción acción de adición de funciones-.

Actividad 3

Alguna de las relaciones " $\oplus$ " o " + ", ¿̇es operación binaria? En caso afirmativo, ¿̇en qué conjunto está definida?

Creemos que, en general, los estudiantes no tendrán problema en indicar que + es operación binaria en $\mathbb{R}$ (o en algún subconjunto de $\mathbb{R}$ ), dado que el tema fue tratado durante el curso de Fundamentos. Sin embargo, interpretar el símbolo " $\oplus$ " como una operación binaria, seguramente les resulte más complicado, debido a que, hasta el momento (en el cuestionario), solo ha aparecido como parte de la notación de la función suma. Creemos que darle una interpretación (autónoma) como operación binaria (como proceso) requiere de un desarrollo importante del esquema de adición de funciones: el estudiante, por analogía con la adición de reales ( + es la operación binaria, definida en $\mathbb{R}$, que a todo par ordenado de números reales $(x, y)$ le hace corresponder el real $x+y$ ), debería poder establecer que $\oplus$ es la operación binaria (objeto), definida en el conjunto $\mathcal{F}$ (objeto) de todas las funciones reales (objeto), que a todo par ordenado $(f, g)$ de funciones reales del conjunto $\mathcal{F}$ (proceso) le hace corresponder (operación binaria como proceso) la función suma $f \oplus g$ (objeto). Para esto es necesario concepciones objeto de operación binaria, conjunto, función y función suma, y concepciones proceso de conjunto y de operación binaria. Debido a que la adición de funciones no había sido trabajada en el curso, creemos que esta última es una respuesta bastante improbable. Otro tipo de respuesta más 
factible, por parte de los estudiantes, podría estar relacionada con indicar que el símbolo " $\oplus$ " se usa para "sumar funciones" (en el mismo sentido en que el símbolo "+" está vinculado con la suma de reales). Esto podría estar señalando un énfasis en aspectos algorítmicos vinculados con la suma de las expresiones analíticas de las funciones, lo cual está relacionado con una concepción acción de adición de funciones según nuestra descomposición genética preliminar.

Actividad 4

¿Un conjunto puede tener como elementos funciones? Justifica.

Según las descomposiciones genéticas de conjunto y de función presentadas más arriba, para responder afirmativamente a esta pregunta es necesario una concepción objeto de función y una concepción proceso de conjunto. Si un estudiante solo tiene una concepción acción o una concepción proceso de función, difícilmente podrá dar una respuesta afirmativa. Por lo tanto, esta pregunta nos daría información sobre el desarrollo del esquema de adición de funciones del estudiante, dado que una concepción proceso de adición de funciones (según la descomposición genética preliminar presentada) requiere concepciones objeto de función y proceso de conjunto.

Actividad 5

Consideremos dos reales $a$ y $b$, y la función $f: \mathbb{R} \rightarrow \mathbb{R}$ que cumple:

\begin{tabular}{|c|c|c|c|c|}
\hline$x$ & -1 & 5 & 1 & $a$ \\
\hline$f(x)$ & -1 & 2 & $a$ & $b$ \\
\hline
\end{tabular}

Consideremos también la función $g: \mathbb{R} \rightarrow \mathbb{R}$ tal que $g(x)=x^{2}+x-1$.

Completa, si es posible, cada columna de la siguiente tabla y explica cómo lo hiciste. Si en algún caso no es posible, indica los motivos.

\begin{tabular}{|c|c|c|c|c|}
\hline$x$ & -1 & 5 & 1 & $a$ \\
\hline$(f \oplus g)(x)$ & & & & \\
\hline
\end{tabular}


En esta actividad hemos optado por no brindar la expresión analítica de $f$ con el objetivo de evitar que los estudiantes, para completar la tabla, hallaran la expresión analítica de $f \oplus g$, en cuyo caso la actividad pasaría a estar centrada en el concepto función y no en el concepto función suma. La resolución de esta actividad requiere una concepción acción de función suma que haga viable encontrar la imagen de cada $x$ según $f$ y $g$ y, luego, sumando dichas imágenes, encontrar la imagen de $x$ según $f \oplus g$.

Actividad 6

Consideremos el conjunto $\mathcal{F}=\left\{f_{1}, f_{2}, f_{3}\right\}$, siendo $f_{1}, f_{2}$ y $f_{3}$ funciones de dominio $\mathbb{R}$ y codominio $\mathbb{R}$, tales que: $f_{1}(x)=x^{3}, f_{2}(x)=-x^{3}$ y $f_{3}(x)=0$.

La relación $\oplus$ de $\mathcal{F} \times \mathcal{F}$ en $\mathcal{F}$, que a cada par ordenado $(g, h)$ de funciones de $\mathcal{F}$ le hace corresponder la función $g \oplus h$, ¿es una operación binaria definida en $\mathcal{F}$ ? Justifica.

Para resolver esta actividad es necesario una concepción objeto de función que permita atribuir un correcto significado al conjunto $\mathcal{F}$ y al par ordenado $(g, h)$. También es necesario una concepción proceso de operación binaria que haga posible considerar todos los pares $(g, h)$ pertenecientes a $\mathcal{F} \times \mathcal{F}$ con la finalidad de encontrar la función suma con la que están relacionados. Una concepción acción de adición de funciones permite encontrar la expresión analítica de $g \oplus h$, cualesquiera sean $g$ y $h$ pertenecientes a $\mathcal{F}$. Luego una concepción acción de conjunto es suficiente para establecer si, en cada caso, $g \oplus h$ pertenece a $\mathcal{F}$.

Actividad 7

Consideremos el conjunto $\mathcal{F}$ formado por todas las funciones de dominio $\mathbb{R}$ y codominio $\mathbb{R}$, y la operación binaria $\oplus$ definida en $\mathcal{F}$, que a cada par ordenado $(g, h)$ de funciones de $\mathcal{F}$ le hace corresponder la función $g \oplus h$. Para que $\oplus$ sea conmutativa se debería cumplir que:

$$
g \oplus h=h \oplus g, \forall g, h \in \mathcal{F} .
$$

La operación binaria $\oplus$ definida en $\mathcal{F}$, ¿̇es conmutativa? Justifica.

Para realizar esta actividad es necesario: (1) Concepciones objeto de adición de funciones y de conjunto para considerar la adición de funciones (objeto) como una operación binaria definida en $\mathcal{F}$ (objeto). (2) Desencapsular el objeto adición de funciones y el objeto conjunto para pasar a sus respectivos procesos, y tener concepciones objeto de función y de función suma. De este modo sería posible considerar la adición de funciones (proceso) como la función que a todo par 
ordenado $(g, h)$ y $(h, g)$ de funciones (objeto) de $\mathcal{F}$ (proceso) le hace corresponder las funciones suma (objeto) $g \oplus h$ y $h \oplus g$, respectivamente. (3) Desencapsular los objetos $g$ y $h$ para pasar a considerar sus respectivos procesos: $g(x)$ y $h(x)$, para todo real $x$. (4) Coordinar dichos procesos a través de la adición de reales, para obtener los procesos $g(x)+h(x)$ y $h(x)+g(x)$, para todo real $x$. (5) Para cada real $x$, se deben considerar las sumas de reales $g(x)+h(x)$ y $h(x)+g(x)$, para luego concluir que son iguales (para cada $x$ real) debido a la propiedad conmutativa de la adición en $\mathbb{R}$. (6) Los procesos $g(x)+h(x)$ y $h(x)+g(x)$, para todo real $x$ (función suma como proceso), se deben encapsular para poder concluir que $g \oplus h$ es igual a $h \oplus g$ (función suma como objeto), cualesquiera sean $g$ y $h$. (7) Encapsular el proceso adición de funciones para establecer que $\oplus$ (objeto) es conmutativa.

\subsubsection{Análisis a priori de las actividades del cuestionario 2}

Como ya mencionamos, luego del análisis de las respuestas al cuestionario 1 y de las entrevistas, comenzó una primera etapa de teorización en la que observamos el fenómeno de identificación. El cuestionario 2 tuvo como finalidad refutar o confirmar la existencia de dicho fenómeno.

A continuación, presentaremos una a una las actividades del cuestionario 2 y su correspondiente análisis a priori.

Actividad 1

Consideremos el conjunto $\mathcal{A}=\left\{h_{1}, h_{2}, h_{3}\right\}$, siendo $h_{1}, h_{2}$ y $h_{3}$ funciones de dominio $\mathbb{R}$ y codominio $\mathbb{R}$, tales que: $h_{1}(x)=x^{3}, h_{2}(x)=-x^{3}$ y $h_{3}(x)=0$.

La relación $*$ de $\mathcal{A} \times \mathcal{A}$ en $\mathcal{A}$, que a cada par ordenado $(f, g)$ de funciones de $\mathcal{A}$ le hace corresponder la función suma de $f$ y $g$, ¿̇es una operación binaria definida en $\mathcal{A}$ ? En caso afirmativo, explica lo más detalladamente posible por qué crees que se cumple la propiedad de unicidad y por qué crees que se cumple la propiedad de clausura. En caso negativo indica, justificando, cuál de las dos propiedades (unicidad y/o clausura) no cumple $*$.

Desde el punto de vista matemático esta actividad es igual a la actividad 6 del cuestionario 1 pero, como mencionamos, hay un cambio deliberado en algunas notaciones con la finalidad de establecer si el fenómeno de identificación estaba vinculado con unas notaciones sugerentes. Con respecto a los cambios de notación, a la relación que a cada par ordenado de elementos del conjunto de 
funciones le hace corresponder la función suma, la hemos representado con el símbolo " $*$ " en lugar del símbolo " $\oplus$ ”. Además, hemos evitado notar la función suma como " $f \oplus g$ ". Estos cambios tuvieron como propósito independizar (desde el punto de vista simbólico) la adición de funciones de la función suma, lo cual era clave para establecer si la identificación entre la adición de funciones y la función suma permanecía más allá de los aspectos simbólicos. Por otra parte, también hubo un cambio en la redacción (respecto a la actividad 6 del cuestionario 1), con la finalidad de que los estudiantes se explayaran es sus respuestas y de esta forma pudiéramos observar cómo ellos concebían el vínculo entre * y la función suma. Con relación al análisis a priori de esta actividad, este coincide con el realizado para la actividad 6 del cuestionario 1.

Actividad 2

Consideremos el conjunto $\mathcal{F}$ formado por todas las funciones de dominio $\mathbb{R}$ y codominio $\mathbb{R}$. La relación $*$ de $\mathcal{F} \times \mathcal{F}$ en $\mathcal{F}$, que a cada par ordenado $(f, g)$ de funciones de $\mathcal{F}$ le hace corresponder la función suma de $f$ y $g$, ces una operación binaria definida en $\mathcal{F}$ ? En caso afirmativo, explica lo más detalladamente posible por qué crees que se cumple la propiedad de unicidad y por qué crees que se cumple la propiedad de clausura. En caso negativo indica, justificando, cuál de las dos propiedades (unicidad y/o clausura) no cumple *.

Según las descomposiciones genéticas presentadas, esta actividad requiere concepciones objeto de operación binaria, conjunto, función y función suma, y concepciones proceso de operación binaria, conjunto y función suma. Para justificar que * es una operación binaria (objeto) definida en $\mathcal{F}$ (objeto), se deben considerar (operación binaria como proceso) dos funciones reales $f$ y $g$ (objeto) cualesquiera del conjunto $\mathcal{F}$ (proceso), y luego la función suma $s$ (objeto) relacionada con $(f, g)$. Como $s$ es una función real (por definición), cualesquiera sean $f$ y $g$, entonces $s$ pertenece a $\mathcal{F}$. Por lo tanto, $*$ cumple clausura $\left({ }^{1}\right)$.

Por otra parte, supongamos que para dos funciones reales $f$ y $g$ (objeto) particulares resultaran dos funciones suma distintas $s_{1}$ y $s_{2}$ (objeto). Como $s_{1}(x)=f(x)+g(x)$ y $s_{2}(x)=f(x)+g(x)$, para todo real $x$ (función suma como proceso), y como la adición en $\mathbb{R}$ cumple unicidad, entonces $s_{1}(x)=s_{2}(x)$, para todo real $x$. Por lo tanto, como $s_{1}$ y $s_{2}$ son funciones (objeto) de dominio y codominio real, $s_{1}=s_{2}$; lo cual nos conduce a una contradicción. Por lo tanto, podemos concluir la unicidad de la función suma, cualesquiera sean $f$ y $g$. Entonces, $*$ cumple unicidad $\left({ }^{2}\right)$. 
Por $\left({ }^{1}\right)$ y $\left({ }^{2}\right)$ se concluye que $*$ (objeto) es operación binaria en $\mathcal{F}$ (objeto).

Actividad 3

Consideremos el conjunto $\mathcal{F}$ formado por todas las funciones de dominio $\mathbb{R}$ y codominio $\mathbb{R}$, y la operación binaria $*$ de $\mathcal{F} \times \mathcal{F}$ en $\mathcal{F}$, que a cada par ordenado $(f, g)$ de funciones de $\mathcal{F}$ le hace corresponder la función suma de $f$ y $g$.

(a) Considera dos funciones particulares pertenecientes a $\mathcal{F}$, por ejemplo:

$$
f: \mathbb{R} \rightarrow \mathbb{R} / f(x)=x^{2}+1 \text { y } g: \mathbb{R} \rightarrow \mathbb{R} / g(x)=x^{3} .
$$

¿Cómo le explicarías a un compañero, utilizando estas funciones particulares para ejemplificar, que * es una operación binaria definida en $\mathcal{F}$ ?

(b) Indica diferencias y similitudes entre "*" y la adición de reales " + ".

Para brindar una posible respuesta a la pregunta de la parte a, se puede encontrar la función suma para estas funciones particulares: $s: \mathbb{R} \rightarrow \mathbb{R} / s(x)=x^{3}+x^{2}+1$; para esto sería necesario una concepción acción de adición de funciones. Luego, como $s$ (objeto) es una función real y es única, entonces el correspondiente (operación binaria como proceso) del par ordenado $(f, g)$ pertenece a $\mathcal{F}$ (proceso) y es único. A continuación, se puede establecer que este mismo razonamiento vale cualesquiera sean las funciones $f$ y $g$ (objeto) y, por lo tanto, * (objeto) es operación binaria en $\mathcal{F}$ (objeto).

Con respecto a la parte $b$, una similitud que se puede establecer es que ambas son operaciones binarias y una diferencia es que están definidas en conjuntos distintos. Poder establecer diferencias y similitudes entre la adición de funciones y otras operaciones binarias exige una concepción objeto de adición de funciones.

\section{CONSIDERACIONES FINALES}

\subsection{CONCLUSIONES}

En nuestra investigación, las descomposiciones genéticas de adición de funciones, conjunto, función, operación binaria y función suma fueron el marco de referencia utilizado (inicialmente) para el análisis a priori de las actividades que conformaron los cuestionarios empleados para recabar información. Este análisis permite justificar la pertinencia de los cuestionarios como herramientas válidas para recabar información con la finalidad de responder la pregunta de investigación y alcanzar los objetivos propuestos para el estudio. 
Como es posible observar en el análisis a priori realizado, las distintas actividades comprometen distintas estructuras mentales vinculadas a la adición de funciones y a conceptos cuyos esquemas son componentes del esquema de adición de funciones. Una concepción acción de conjunto es necesaria en la actividad 6 del cuestionario 1 (de aquí en más, salvo que se indique expresamente, se estará haciendo referencia a actividades del cuestionario 1) y en la actividad 1 del cuestionario 2 (que son iguales desde el punto de vista matemático). Una concepción proceso de conjunto es necesaria en las actividades 3, 4, 7, 2/2 y 3a/2 (agregamos, luego del número de la actividad, una barra seguida de un 2 para indicar que la actividad corresponde al cuestionario 2). Por otra parte, una concepción objeto de conjunto es necesaria en las actividades 3, 7, 2/2 y 3a/2. Una concepción acción de función no es necesaria en ninguna de las actividades, en cambio es necesaria una concepción proceso en la actividad 7 , y una concepción objeto en las actividades 3, 4, 6, 7, 1/2, 2/2 y 3a/2. Con respecto a la función suma, las actividades 1 y 5 están vinculadas a una concepción acción, la 1 y la 7 a una concepción proceso y las actividades 1, 2, 3, 7, 2/2, 3a/2 a una concepción objeto. Pasando ahora al concepto operación binaria, tenemos que en la actividad 2 se podría poner en juego una concepción acción, en las actividades 3, 6, 2/2, 3a/2 es necesaria una concepción proceso, y las actividades 2, 3, 2/2,3a/2 están relacionadas a una concepción objeto. Por último, en relación con la adición de funciones, las actividades 2, 3, 6 y 3a/2 están vinculadas a una concepción acción, en la 7 es necesaria una concepción proceso y en las actividades 7 y 3b/2 una concepción objeto.

El análisis a priori realizado permite objetivar y brindar racionalidad a las exigencias cognitivas involucradas en las resoluciones de las distintas actividades que conformaron los cuestionarios utilizados para recabar información (por ejemplo, la actividad 5 requeriría únicamente una concepción acción de función suma -lo que la convierte en una actividad de muy baja exigencia cognitiva-; en cambio, la actividad 7 es la más exigente desde el punto de vista cognitivo: requiere concepciones proceso y objeto de conjunto, función, función suma y adición de funciones). Esto no solo nos permite justificar la pertinencia de las actividades para los propósitos de nuestra investigación, sino además aseverar que las actividades contienen un amplio margen de dificultad que nos permite obtener información variada en relación con el rendimiento matemático de los estudiantes:

La idea es obtener datos que muestren un rango amplio del rendimiento matemático sobre diferentes tareas matemáticas que permita comparar el pensamiento de 
los estudiantes que tuvieron dificultades con el pensamiento de los estudiantes que tuvieron éxito. Estas diferencias permiten a los investigadores determinar si las construcciones mentales requeridas por el análisis teórico explican las diferencias en el rendimiento o si se requieren otras construcciones mentales no consideradas por el análisis teórico. (Arnon et al., 2014, p. 95)

En resumen, el análisis a priori de las actividades de los cuestionarios nos permite afirmar que: (1) los cuestionarios (utilizados para recabar información) están en consonancia con la pregunta de investigación y con los objetivos propuestos para el estudio y (2) las actividades tienen el potencial de capturar un rango amplio del rendimiento matemático de los estudiantes.

Por otra parte, el análisis a priori, utilizando como marco de referencia la descomposición genética, puede resultar de utilidad a los docentes para comprender las exigencias - desde el punto de vista cognitivo- involucradas en la resolución de ciertas actividades. El conocimiento de dichas exigencias puede posicionarlos mejor a la hora de tomar decisiones que colaboren con el aprendizaje de sus estudiantes.

La descomposición genética presentada en este trabajo evidencia que un objeto matemático por muy «simple» que parezca o que algunos libros de texto presenten de forma trivial, implica un conjunto de construcciones mentales y mecanismos de construcción que los estudiantes requieren desarrollar para lograr su comprensión... (Jaimes, Chaves y Vargas, 2017, p. 77)

Por último, consideramos que las descomposiciones genéticas preliminares de adición de funciones y de función suma presentadas en este artículo constituye una contribución a la literatura.

\subsection{DESCRIPCIÓN GENERAL DE LA INVESTIGACIÓN Y COMENTARIOS PRELIMINARES SOBRE SUS RESULTADOS}

Nuestra investigación comenzó con una descomposición genética preliminar de adición de funciones. Debido a los propósitos de nuestro estudio, creímos necesario contar además con descomposiciones genéticas de conceptos cuyos esquemas son componentes del esquema de adición de funciones. Un análisis de la adición de funciones como proceso nos permitió establecer que los esquemas de conjunto, función, operación binaria y función suma son componentes del 
esquema de adición de funciones. No fue necesario realizar descomposiciones genéticas de conjunto, función y operación binaria, dado que ya existían en la literatura (Arnon et al., 2014); en cambio elaboramos una descomposición genética preliminar de función suma.

Las descomposiciones genéticas mencionadas sirvieron como marco de referencia, inicialmente, para realizar un análisis a priori de las actividades de los cuestionarios que utilizamos para recabar información y, luego, para analizar las respuestas de los estudiantes a las preguntas planteadas en los cuestionarios y en las entrevistas que realizamos.

Cuando comenzamos el análisis, nos encontramos con cierta regularidad: muchos estudiantes identificaban la adición de funciones con la función suma, considerándolas como si fueran el mismo concepto matemático. Luego advertimos que esta identificación era un caso particular de una más general: la identificación de una relación con los elementos de su conjunto de llegada. A este fenómeno lo llamamos identificación y lo explicamos a través de la teoría APOE.

Después de haber explicado el fenómeno de identificación, analizamos la información recogida en relación con las estructuras mentales (acción, proceso y objeto) vinculadas a la adición de funciones y a la función suma, con la finalidad de validar o refinar las descomposiciones genéticas preliminares. Este análisis nos condujo a validar la descomposición genética preliminar de adición de funciones y a refinar la de función suma.

Una vez que contamos con dichas descomposiciones genéticas (la validada y la refinada), las utilizamos como marco de referencia para analizar las respuestas de los estudiantes con el propósito de alcanzar los objetivos propuestos para esta investigación:

- Establecer si es suficiente -para construir el esquema de adición de funciones- abordar en la enseñanza, en forma aislada, conceptos como conjunto, función y operación binaria.

- Establecer fenómenos inherentes a la construcción del esquema de adición de funciones.

Con respecto al primero de los objetivos, hemos encontrado elementos suficientes para apreciar la complejidad y las exigencias cognitivas presentes en la construcción del esquema de adición de funciones. Por lo tanto, consideramos que no es suficiente -desde el punto de vista de la enseñanza-centrar la atención en cómo los estudiantes aprenden, en forma aislada, conceptos como 
conjunto, función y operación binaria, es necesario, también, trabajar intencionalmente en la construcción de vínculos entre los mismos. En relación con el segundo de los objetivos, el análisis nos permitió advertir, además del fenómeno de identificación, la existencia de otros dos fenómenos: el de interacción/interferencia de esquemas y el de enmarcación, pero el reporte de los mismos excede los propósitos de este artículo.

\section{REFERENCIAS}

Arnon, I., Dubinsky, E., Cottrill, J., Oktaç, A., Roa-Fuentes, S., Trigueros, M., y Weller, K. (2014). Apos Theory. A Framework for Research and Curriculum Development in Mathematics Education. Springer.

Dubinsky, E., y McDonald, M. A. (2001). APOS: A constructivist theory of learning in undergraduate mathematics education research. En D. Holton (Ed), The Teaching and Learning of Mathematics at University Level: An ICMI Study (Vol. 7, pp. 273-280). Kluwer Academic Publishers.

Eisenberg, T. (1991). Functions and associated learning difficulties. En D. Tall (Ed.). Advanced Mathematical Thinking (pp. 140-152). Kluwer Academic Publishers.

Jaimes Contreras, L. A., Chaves Escobar, R. F., y Vargas Hernández, J. (2017). La descomposición genética como herramienta para matemáticos, ingenieros y licenciados en la enseñanza del cálculo: Investigación en educación matemática. Revista Boletín Redipe, 6(9), 73-78.

Roa-Fuentes, S., y Oktaç, A. (2012). Validación de una descomposición genética de transformación lineal: un análisis refinado por la aplicación del ciclo de investigación de la teoría APOE. Revista Latinoamericana de Investigación en Matemática Educativa, 15(2), 199-232.

Trigueros, M. (2005). La noción de esquema en la investigación en matemática educativa a nivel superior. Educación Matemática, 17(1), 5-31. 